\title{
Über das „Lob der Kahlheit“ des Synesios von Kyrene mit trichologischem Rückblick in die Antike sowie Ausblick in Gegenwart und Zukunft
}

\author{
On the „Praise of Baldness“ by Synesius of Cyrene with a Trichological Look Back to Antiquity and a Look \\ Forward into the Present and Future
}

Autor

Institut

\section{R. Möhn}

Arzt für Hautkrankheiten und Allergologie, Hanau

\section{Bibliografie}

DOI http://dx.doi.org/

10.1055/s-0030-1256718

Online-Publikation: 25. 8. 2011

Akt Dermatol 2011; 37:

464-471 @ Georg Thieme

Verlag KG Stuttgart · New York ISSN 0340-2541

\section{Korrespondenzadresse}

Dr. med. Rudolf Möhn

Arzt für Hautkrankheiten

und Allergologie

Philippsruher Allee 35

63454 Hanau

rmoehn@unitybox.de

\section{Zusammenfassung \\ $\nabla$}

Zunächst wird auf Leben und Werk des Synesios von Kyrene eingegangen, gefolgt von wichtigen Aspekten der Haartracht allgemein. Sodann werden die wichtigsten Argumente des Synesios zum Lob der Kahlheit aus Biologie, Kosmologie, der griechischen Mythologie und der allgemeinen Zeitgeschichte vorgetragen. Mit zum Teil sophistischen Methoden werden die Vorzüge der Kahlheit besonders am Beispiel des Halbgottes Asklepios bzw. seines ägyptischen Vorbildes Imhotep und an dem kahlköpfigen Philosophen Sokrates im

„Von den Haaren konnte gezeigt werden, dass sie Ausdruck von Unvernunft, tierischer Natur und Teil der Front gegen das Göttliche sind.“

Synesios von Kyrene, Lob der Kahlheit, Übersetzung Werner Golder, ([1], S. 81)

\section{Einleitung \\ $\nabla$}

„Daher fühlte ich mich, gleich, nachdem das Verhängnis über mich hereingebrochen war und die Haare zu schwinden begannen, wie mitten ins Herz getroffen. Als das Schicksal dann aber seinen Lauf nahm und ein Haar nach dem anderen ausfiel, manchmal auch zwei auf einmal oder ein ganzes Büschel, und der Krieg auf meinem mehr oder weniger geplünderten Kopf so richtig wütete, da glaubte ich wirklich ärger leiden zu müssen als einst die Athener unter dem von Archidamos befohlenen Kahlschlag in den Wäldern von Acharnai. Es dauerte nicht lange, da sah ich aus wie einer von den ungepflegten Euböern, die nach der Schilderung in der Ilias nur am Hinterkopf behaart sind und in dieser Aufmachung gegen Troja zu Felde zogen.“(Übersetzung Werner Golder, [1], S. 7)
Kontext zu den mythologischen Figuren Dionysos und Silenos dargelegt. Die Ikonografie dieser Figuren und eigene Betrachtungen dazu führen zu verblüffend bestätigenden Ergebnissen. Auf die konträre, in seinem Erstlingswerk „Die Geburt der Tragödie“" von Friedrich Nietzsche dargelegte Beurteilung des Dionysos und Sokrates und ihre Analogie bei der trichologischen Betrachtung wird hingewiesen. Im Ganzen zeigt sich, dass Haartracht und Wertigkeit der Glatze im Laufe der Geschichte einer ständigen Wandlung unterliegen, für die Zukunft wird eine gesellschaftliche Aufwertung der Kahlköpfigkeit erwartet.

\section{Synesios von Kyrene und sein Werk}

$\nabla$

Mit soviel Selbstironie beginnt das „Lob der Kahlheit" (phalakras enkomion, lat. calvitii encomium), ein Werk aus der Feder des griechischsprachigen und der Tradition der griechischen Klassiker verpflichteten frühchristlichen Philosophen Synesios von Kyrene (aus der Kyrenaika, heute Bengasi, Libyen), um 370-412 n.Chr. Es endet mit der als Motto vorangestellten, wahrhaft gewalttätigen Erkenntnis über die Natur der Haare. Synesios war Schüler der später von fanatischen Christen umgebrachten platonischen Philosophieprofessorin (!) Hypatia in Alexandria $(\diamond$ Abb. 1) und zeitlebens dem Platonismus verbunden, obwohl er später als eher widerwilliger Christ sogar Bischof von Ptolemais wurde.

Von seinen Werken sind Hymnen, Briefe und verschiedene Abhandlungen erhalten geblieben u.a. „Dion“, in der er sich mit der Lebensweise des berühmten Redners Dion Chrysostomos (Dion von Prusa, heute Bursa, Türkei) beschäftigt. Dieser Dion hatte auch eine (nicht erhaltene) Schrift mit dem Titel „Lob des Haupthaares“ verfasst, der Synesios das „Lob der Kahlheit“ entgegensetzt. Es ist überraschend, mit wie viel Zitaten von Homer bis zu den Autoren der klassischen griechischen 


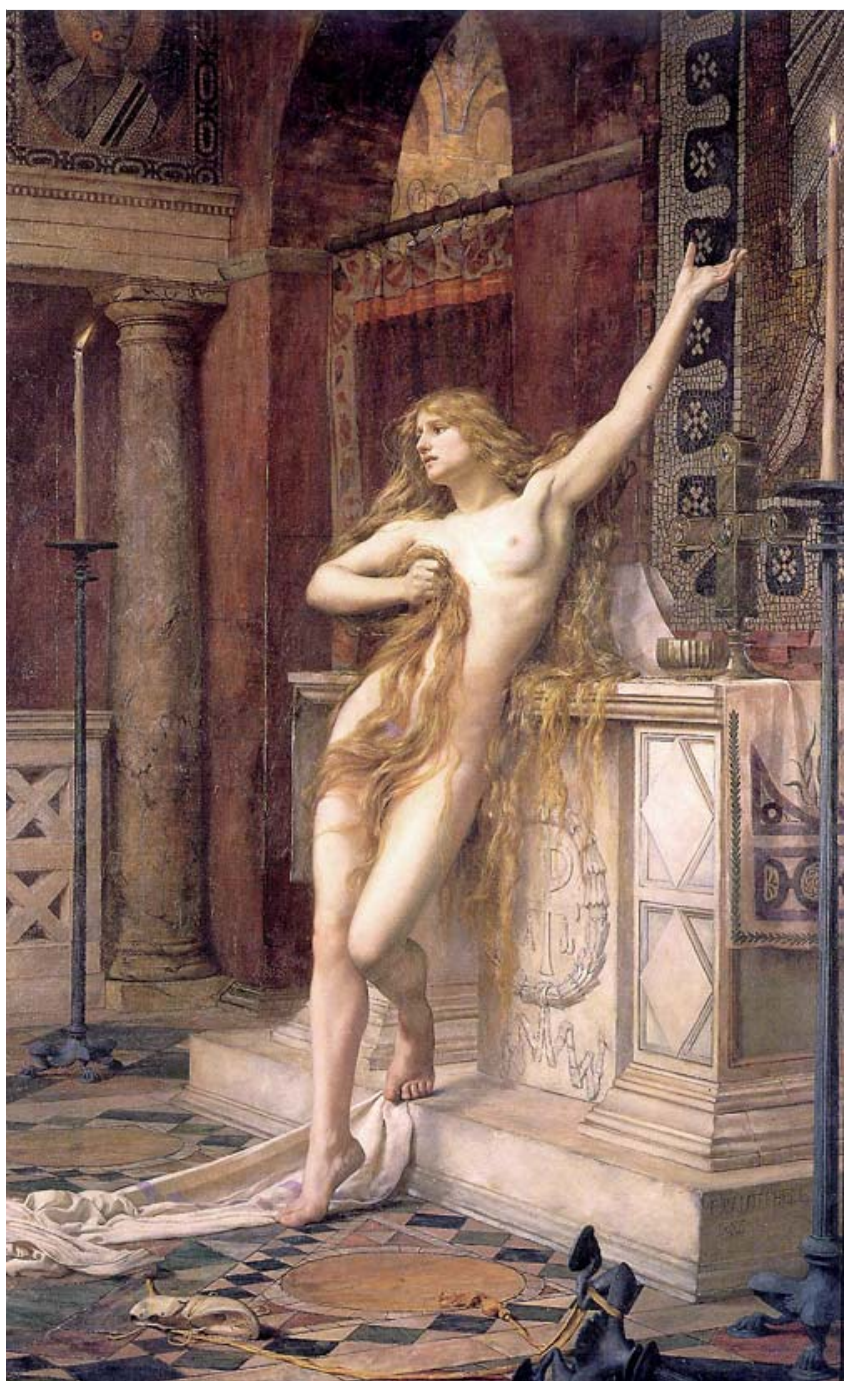

Abb. 1 Hypatia, Philosophie- und Mathematikprofessorin in Alexandria (ca. 355-415 n. Chr.), Lehrerin des Synesios von Kyrene vor ihrer Ermordung in einer christlichen Kirche. Retrospektives, idealisierendes Gemälde von C. W. Mitchell, 1885, Laing Art Gallery, Newcastle. „Teuflisch“ schöne, junge Frau mit „ungöttlichem“ wallendem Hexenhaar (Bild: Wikipedia).

Zeit wie Herodot, Platon oder Xenophon er sein Werk über ein scheinbar banales Thema angereichert hat. Eine Abbildung von ihm und seiner Kahlheit konnte ich nicht finden.

\section{Geschichte der Haartracht}

\section{$\nabla$}

Bevor wir näher auf sein Werk eingehen, jedoch einige Vorbemerkungen zur Haartracht und zu Haarritualen ganz allgemein. Schönes, volles, vielleicht zusätzlich gelocktes oder mit Zopf geschmücktes Haar war und ist in allen Kulturkreisen ein Attribut

- männlicher und weiblicher Jugend und Schönheit, bei Frauen auch Verführungskunst

- des kraftvollen und strahlenden Kämpfers und Kriegshelden (Beispiel Achilles, Samson)

- des Machtanspruchs im politischen Bereich (coma caesarea der römischen Kaiser)

- der Überlegenheit in der geistigen Sphäre (z. B. Perückenkult im Barock)
- zur Betonung von Freiheit und Unabhängigkeit, vor allem im europäischen Mittelalter.

Im sakralen Bereich waren Haarrituale mit Rasur oder Teilrasur und Belassung eines Zopfes weit verbreitet, so auch die in der griechischen Antike bekannte Theseis, benannt nach dem Helden Theseus, der sein Haar bis auf den charakteristischen Zopf in Delphi geopfert hatte. Dasselbe taten die Helden von Troja, wenn Sie einem toten Mitstreiter bei der Trauerfeier die Haare ins Bestattungsfeuer nachwarfen. Ihr Haar im Tempel der Aphrodite zu Thmuis (Nildelta) geopfert hatte auch Berenike II, die Gemahlin von Ptolemaios III Euergetes 246 v. Chr. Nachdem das Haar verschwunden war, wurde erklärt, dass die Göttin es unter die Sternbilder als „coma Berenicis“ transfiguriert habe. Ein typisches Beispiel für den in der Antike so häufigen und auch bei Synesios vorkommenden astral-kosmologischen Bezug der Haartracht. Das berühmte Mosaik der Berenike zu Thmuis müsste Synesios eigentlich gekannt haben, in seiner Schrift berichtet er aber nichts davon. Ähnliche Haaropferungen aus traurigen oder freudigen Anlässen gab es auch bei vielen anderen Völkern, z.B. dem ägyptischen Jugendzopf und dem vorchristlichen polnischen Haarritual Postrzyzyny, das später für die Geschichte des Weichselzopfes so bedeutend wurde [2,3]. Das Haar war demnach eines der kostbarsten Güter, das der Mensch hatte, seine Pflege besonders wichtig und diese hatte selbst magisch-mystische Bedeutung, denn nicht anders ist z.B. das von Herodot ([1] S. 15) berichtete und von Synesios zitierte merkwürdige Verhalten der spartanischen Helden vor der Schlacht bei den Thermopylen (480 v.Chr.), in der sie alle fielen, zu erklären. Am Kampfplatz angekommen, hatten sie nichts Wichtigeres zu tun, als sich ihr Haar sorgfältig zu kämmen, wo man doch hätte annehmen können, dass eine möglichst wild zerzauste Mähne einen viel größeren Eindruck auf den Gegner mache. Diese Episode mag die Brücke zu einer näheren Beschäftigung mit Synesios bilden, der diesen ästhetischen Verfeinerungen der Haartracht und dem Haaropferungskult den groben Klotz des „Lobes der Kahlköpfigkeit" entgegensetzt. Wie das? Seine wichtigsten, unverkennbar vom Platonismus beeinflussten, halb-metaphysischen, astralen, symbolistischen sowie kosmologischen, biologischen und historischen Argumente werden hier vorgetragen.

\section{Argumente für die Kahlköpfigkeit}

$\nabla$

1. Der Mensch ist die Krone der Schöpfung, unter allen Lebewesen das Nackteste und nur noch an wenigen Stellen behaart. Je weniger Haare er hat, desto mehr unterscheidet er sich vom dümmsten aller Tiere, nämlich dem Schaf, dem die Haare nicht einzeln, sondern in dichten Büscheln wachsen. Die für die Entfaltung der Geistigkeit wichtigsten Organe des Menschen sind die Sinnesorgane, und unter ihnen sind am vornehmsten die völlig kahlen, aber göttlichen Augen, was bei den oft durch Haare versperrten Ohren (oder der Nase) schon nicht mehr so der Fall ist. Haare also stehen im Antagonismus zur geistigen Funktion des Menschen, logisch also, dass der völlig Kahle das geistvollste und göttlichste Lebewesen auf Erden ist ([1], S. 20-24).

2. Kosmologische Gründe: Blüten, Stängel, Blätter, Wurzeln, haarartige Grannen usw. sind vergängliche Bestandteile der Pflanzen, gewissermaßen Spielzeuge der Materie, wichtig und essenziell sind nur die völlig nackten und kahlen, aber göttlichen Samen und Früchte z.B. Getreidekörner, Nüsse, geschälte Hülsenfrüchte usw., aus denen jederzeit wieder neues Leben hervorgehen kann. Wie also die Frucht ihren überflüssigen Zierrat aus Schalen, Blü- 
ten und Blättern abgeworfen hat, so hat erst in einem Kahlkopf, der das überflüssige Haarkleid abgeworfen hat, der Verstand als etwas Gottähnliches endgültig seine Wohnung genommen. „Wenn du einen Kopf siehst, der vollkommen haarlos ist, dann kannst du darauf vertrauen, dass dort der Geist eingezogen ist, dann kannst du den Kopf für einen Tempel des Gottes halten“ ([1], S. 35). Logische Konsequenz, dass man sich auch die Götter, besonders Zeus, im Gegensatz zu den üblichen künstlerischen Darstellungen mit wallendem Haar z. B. bei Homer und Phidias mit völlig nacktem, kugelförmigem Kopf als astrale Erscheinungen vorstellen muss, angelehnt an Platon, Phaidros, $246 \mathrm{~d}$, nach ([1], S. 39).

Sehr platonisch sind auch die Ausführungen zu den Seelen, die sich in den himmlischen Sphären von kugelförmigen Sternen, Sonne und Mond niedergelassen haben und im Gegensatz zu den einzig behaarten Himmelskörpern, den Kometen (kome, das Haar), völlig nackt sind. Auf Erden lassen sich die wertvollsten Seelen in den kugelförmig, nackten Köpfen nieder, und nur die vergleichsweise schlichten Exemplare in den behaarten Köpfen (nach [1], S. 36-39).

3. Der Kahlkopf und das Licht: Hier bezieht sich Synesios auf Homer, der in der Odyssee beschreibt, wie der Heimkehrer Odysseus von einem der aufdringlichen Freier Penelopes, Eurymachos, wegen seiner Kahlköpfigkeit geschmäht wird und unfreiwillig richtig hinzufügt, man brauche ja gar kein Licht im Hause, da aus seinem Kopf ein Leuchten wie von Fackeln komme (nach [1], S. 47). Synesios abstrahiert sofort, indem er das Licht, göttlichen Ursprungs, der Kahlheit, die Dunkelheit aber der Behaarung zuordnet.

4. Griechische Geschichte: Viele griechische Persönlichkeiten, in der breiten Masse die Priester, Wahrsager, Tempeldiener, Lehrer, Pädagogen, Feldherren vor allem, aber wahre Koryphäen des Geistes wie die Philosophen Sokrates ( $\bullet$ Abb.2) und Diogenes ( $\bullet$ Abb. 3) sind kahl gewesen.

Hier scheint der apodiktische Satz des Synesios, dass „einer nur dann weise ist, wenn er eine Glatze hat und umgekehrt nicht weise sein kann, wenn er keine hat", am ehesten richtig zu sein. Ebenso auch, „dass dort der Verstand einzieht, wo die Haare weichen, und dort die Haare wachsen, wo der Verstand sich davonmacht". Andere große Persönlichkeiten wie die berühmten Kriegshelden Hektor, Achilles und Menelaos waren zwar gut und - von Synesios stark bevorzugt blond - behaart, aber man muss das ihrer Jugend zugute halten, denn mit zunehmender Reife und Weisheit wären auch sie in den Club aufgenommen worden ([1], S. 71-81).

\section{Behaarung bei Dionysos und indirekte Kahlheit bei Asklepios \\ $\nabla$}

Einer näheren Betrachtung unterzieht unser Autor Dionysos, den Gott des Weines und des orgiastischen Rausches. Die Mitglieder einer dionysischen Festgesellschaft tragen dichtes eigenes und sogar Fremdhaar, bevorzugt vom Hirschkalb, schneiden Grimassen, führen unanständige Tänze auf und geben sich der Trunkenheit und sexuellen Ausschweifungen hin, verhalten sich also dumm und roh. Hingegen sollte sein von Zeus bestellter Lehrer und Zuchtmeister Silenos zu Mäßigung und Besonnenheit mahnen, und immer als Glatzkopf erscheinen. Die Figur des halbgöttlichen Asklepios bereitet Synesios Schwierigkeiten, denn irgendwelche dionysische Dummheiten wird man ihm nicht so leicht

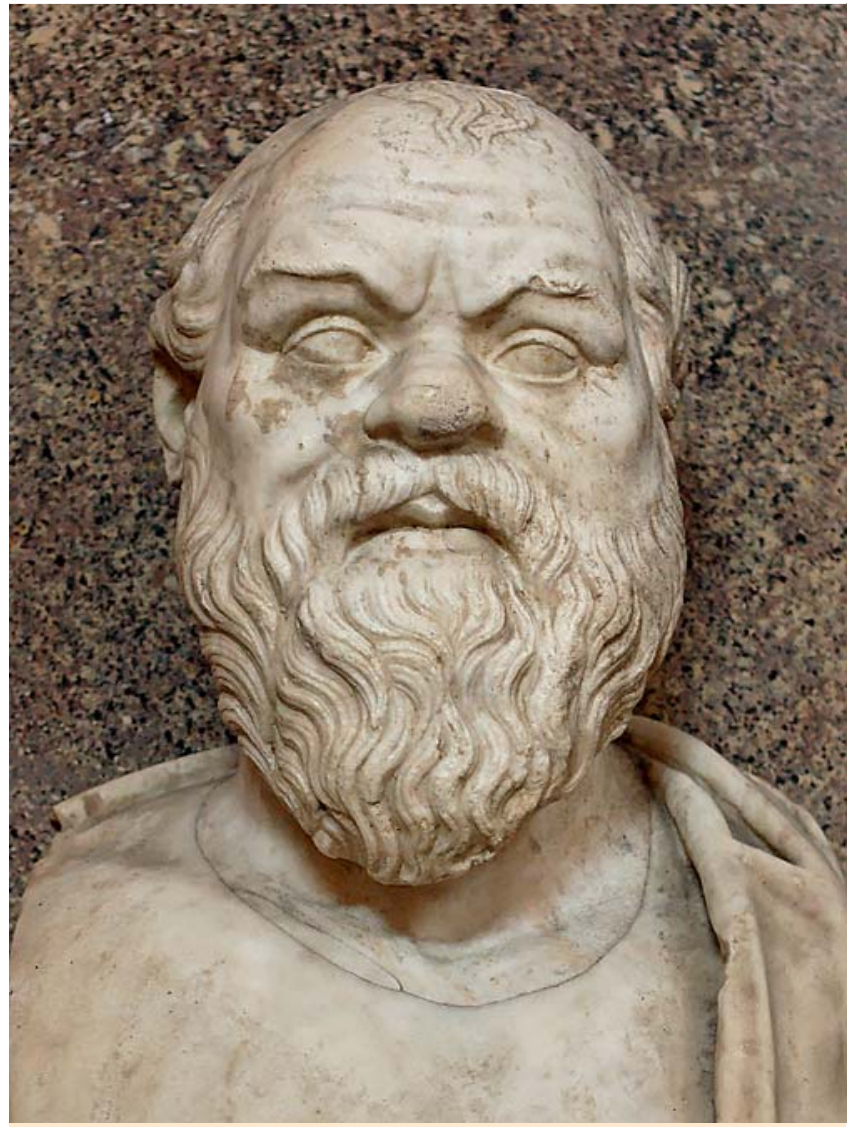

Abb. 2 Büste des Sokrates, vermutlich Kopie eines zu Lebzeiten des Sokrates oder kurz danach entstandenen griechischen Originals, Rom, Vatikanische Museen (Bild: Wikipedia, Marie-Lan Nguyen).

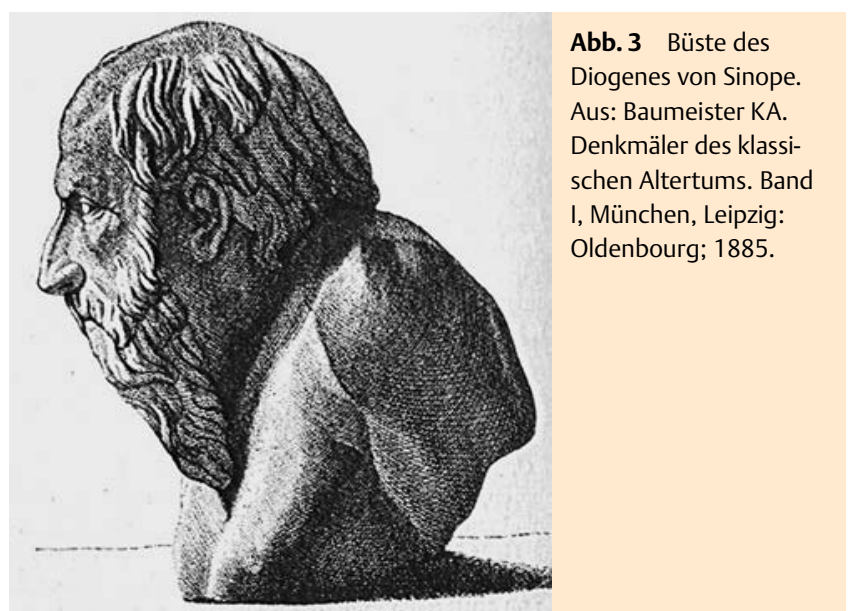

nachweisen können, und trotzdem wird er nicht als Glatzkopf, sondern als gut behaart dargestellt ( $\bullet$ Abb. 4).

Hier behilft sich Synesios mit der Ausflucht, dass nach Thukydides „den Griechen die Suche nach der Wahrheit nicht die Mühe wert ist", (nach [1], S. 43) und beruft sich auf dessen Attribut, die kahle Mörserkeule und auf sein ägyptisches (bei ihm namentlich nicht genanntes) Vorbild Imhotep, gr. Imuthes ( $\bullet$ Abb. 5), der stets kahlköpfig dargestellt werde. 


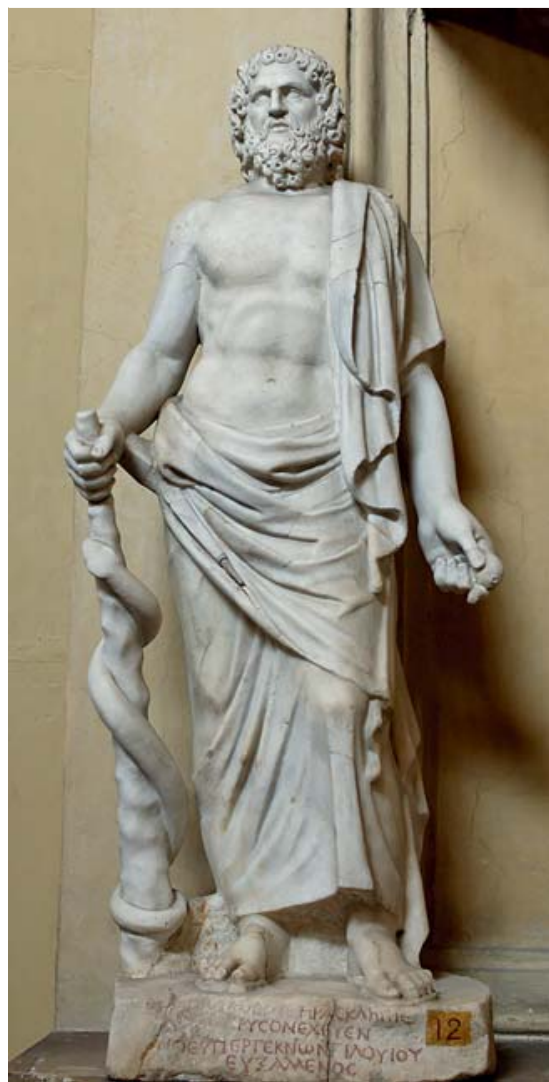

Abb. 4 Asklepios, der Gott der Heilkunst. Obwohl Synesios eigentlich von ihm eine Glatze verlangt, trägt er volles Haar und zeigt nur indirekte Merkmale der Kahlheit z. B. die Mörserkeule, Rom, Vatikanische Museen (Bild: Wikipedia, Marie-Lan Nguyen).

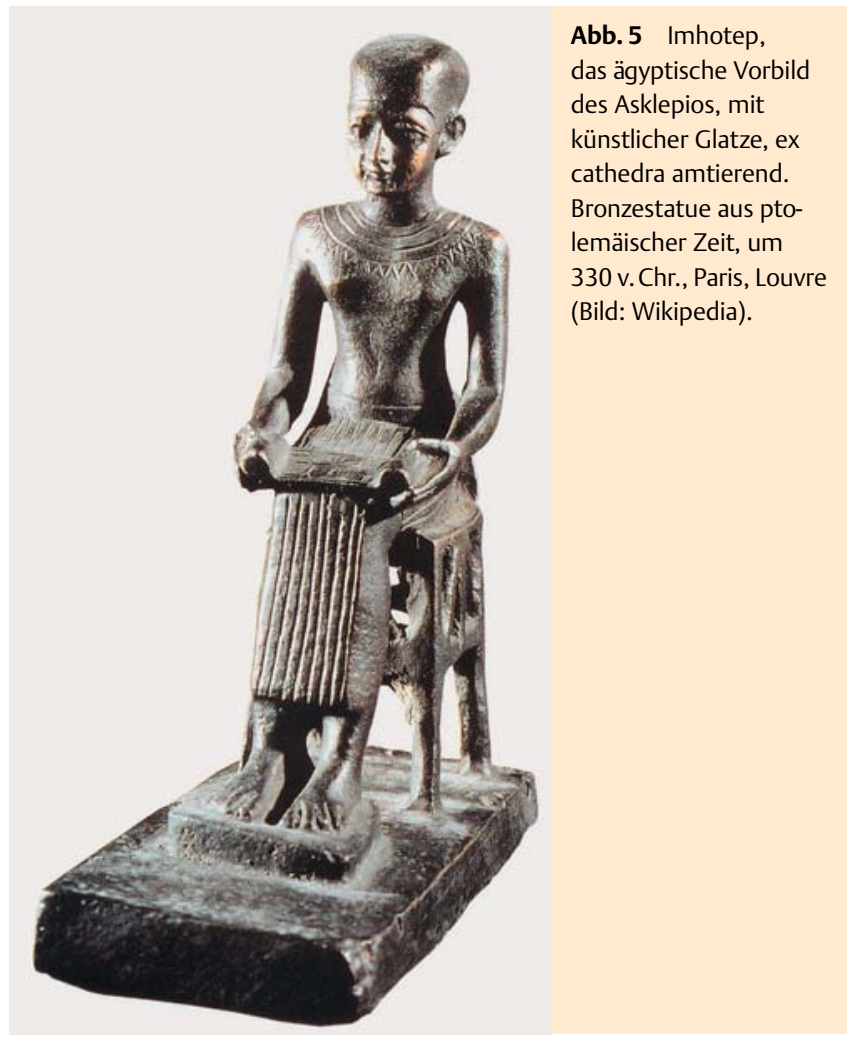

\section{Die androgenetische Alopezie der Frau}

\section{$\nabla$}

Die abschließenden Ausführungen des Synesios zur Haartracht und meist nicht vorhandenen Glatzköpfigkeit von Olympiern, Kriegshelden, Ehebrechern und anderen Gruppen, vor allem aber auch der Frauen erscheinen mir wenig essenziell, sodass sie hier vernachlässigt werden können (nach [1], S. 49, 77, 81). Es wäre aber sicher in seinem Sinn als Schüler einer Frau, wenn ich an die meistens nicht totale, aber keineswegs seltene, starke androgenetische Alopezie der Frau erinnere, die in eindrucksvoller Weise und in vielen Kulturen noch betonter als bei den Männern als ein Attribut der Weisheit erscheint. Dem Opernfreund werden hier vielleicht die stets mit sehr hoher Stirn dargestellten allwissenden und „weltweisen“ Nornen (lat. Parzen, griech. Moiren) aus Wagners „Götterdämmerung“ in den Sinn kommen.

\section{Eigene Gedanken über Asklepios, Imhotep, Silenos, Dionysos und Sokrates $\nabla$}

Die Bemerkung vorausgesetzt, dass Abbildungen aus der „vorfotografischen“ Zeit der Antike nur sehr bedingt und schon gar nicht bei mythologischen Figuren die wirkliche Realität widerspiegeln, sondern Ausdruck kulturgeschichtlicher Überlieferungen oder des Zeitgeschmackes sind und bei viel nachgeborenen Künstlern zeitgenössische Rückprojektionen auf Archetypen in archaischer Zeit zeigen, bin ich bei weiterem „trichologischem“ Nachdenken über die in der griechischen Mythologie und Geistesgeschichte so bedeutenden und auch bei Synesios gesondert behandelten Figuren wie Asklepios bzw. Imhotep, Silenos, Dionysos und Sokrates zu überraschenden Ergebnissen gekommen. Auf - Abb. 4 erscheint der hervorragende Arzt Asklepios, der sagenhafte Sohn des Zeus und der Koronis, als athletischer Mann mittleren Alters mit starkem Haar- und Bartwuchs. Er hat die von der Asklepiosschlange umwickelte und von Synesios als „kahl“ bezeichnete Mörserkeule, den Äskulapstab (in anderen Abbildungen aber oft nur einen simplen Holzstock), ein Sinnbild für den Heilkundigen, in der rechten Hand. Dieser „kahle“ Stab soll in trichologischer, aber recht sophistischer Agogik seine Weisheit repräsentieren (nach [1], S. 43). Merkwürdig nur, dass Synesios auf die abgesehen von den Schuppen ebenso „nackte“ und „kahle“ Schlange, immerhin ein belebtes Wesen, das im Rufe der hinterhältigen List und Weisheit steht, nicht zu sprechen kommt und sie nicht als deren Symbol bezeichnet. Hier wäre doch eine Parallele sehr viel angebrachter. Auf mehr oder weniger behaarte Tiere, z.B die o.g. Schafe, aber auch die Jagdhunde ist er ja auch sonst eingegangen. Von diesen sollen nach dem Hundebuch des Xenophon die am wenigsten behaarten die Besten sein (nach [1], S. 23).

\section{Imhotep und die Vorliebe der Ägypter für Glatzen $\nabla$}

Das ägyptische Vorbild des Asklepios Imhotep (Imuthes, - Abb. 5) war ein hoher Würdenträger, Universalgelehrter, Baumeister und Arzt, der um 2700 v. Chr. zu Zeiten der 3. Dynastie gelebt hat und später im neuen Reich als Heilgott verehrt wurde. Obwohl Synesios voller Begeisterung die Ägypter als konsequente Verfechter der Rasur - bei religiösen Zeremonien würden sie nicht einmal die Augenbrauen stehen lassen - und der ständig dem Sonnenlicht ausgesetzten Glatze preist, welche sogar die im Kriege so wichtige Qualität von Eisen erreiche (nach [1], S. 53), 
übersieht er doch, oder konnte es vielleicht nicht wissen, dass die gängigen Abbildungen den göttlich verehrten Imhotep nicht mit einer natürlichen, sondern einer künstlichen, durch eine eng anliegende Kappe oder gar einen eigens angelegten, turbanartigen Verband vorgetäuschten Glatze zeigen. Nur so vorbereitet und vom Volke anerkannt, konnte in sakraler oder amtlicher Zeremonie „ex cathedra“ gesprochen werden. Sieht man sich ein wenig in der ägyptischen Ikonografie und Literatur um, wird man aber sehr schnell merken, dass auch andere künstliche Haartrachten wie die Perücke und sogar der künstliche, auch der Pharaonin Hatschepsut zugeordnete Bart sehr wichtig waren. Die Glatze scheint nur den obersten geistlichen und weltlichen Autoritäten vorbehalten gewesen zu sein.

Einen Höhepunkt erreicht ihre Wertschätzung in der Zeit der Sonnenverehrung des Ketzerkönigs Echn-Aton. A Abb. 6 zeigt den berühmten, seinen Sonnengott anbetenden Pharao EchnAton mit der ungeschmückten, einer künstlichen Glatze vergleichbaren Krone Oberägyptens, die basale Krone Unterägyptens ist kaum noch zu erkennen.

Die aus der systematisch Inzucht betreibenden Echn-Aton-Sippe bekannte monströse dolichozephale, immer kahle Schädeldeformation wird durch die Krone überbetont und erhebt einen kosmologischen Anspruch als von der Sonne Atons maximal beleuchteter und größenmäßig ebenbürtiger Himmelskörper, einem kahlen Planeten. Auch hier also wieder der für die Antike so typische, schon oben erwähnte astral-kosmologische Bezug der Kahlheit.

Die Vorliebe der Ägypter für künstliche Frisuren war auch ein Thema für Thomas Mann in seinen „Josephsromanen“. In dem Teil „Joseph der Ernährer“ kommt der bedächtige, sachlich-ruhige und kluge ägyptische Amtmann des Gefängnisses, Arzt und spätere Hausverwalter Josephs Mai-Sachme vor, den der Dichter in oft geübter Art und wie ein Bildhauer ganz konkret nach einem lebenden Menschen modellierte. In diesem Fall war sein Vorbild der ihm freundschaftlich verbundene deutsch-amerikanische, kahlköpfige Dermatologe und fast vergessene Schriftsteller Martin Gumpert ([4], S. 31). Schamhafterweise lässt er ihn in einer Erstbeschreibung zwar mit einer Perücke auftreten, wohl aber begleitet von seinem Bannerträger „Blankschädel“, einem Adepten des wolfsköpfigen Gottes Wewaret [5].

In dem vor 50 Jahren berühmten Film „Die zehn Gebote“ spielte der seinerzeit sehr bekannte, unter einer Alopezia areata totalis leidende Schauspieler Yul Brynner den Pharao. Geht man mit dem Stichwort „Imhotep“ ins Internet, fällt einem sofort eine ganze Galerie von Glatzköpfen auf, die sich alle auf ihn beziehen.

\section{Der Helm des Perikles}

$\nabla$

Die künstliche Glatze ist aber auch für die griechische Antike und Synesios nicht nur für geistige, sondern auch für kriegerische Anlässe von Bedeutung gewesen, und zwar in Form des Helmes. Wie schon an der Krone Oberägyptens in $\bullet$ Abb. 6 gezeigt, liefert „auch der Helm in seiner Urform ein genaues Abbild der Glatze und ist der Ausrüstungsgegenstand der Soldaten, der den meisten Schrecken einflößt“ (nach [1], S. 67), und „die glatte und glänzende Oberfläche des Helmes ist es wohl, die, wie eine Glatze wirkt und Schrecken einflößt“" (nach [1], S. 69). Dazu passt sehr gut die berühmte Büste des Perikles, die den griechischen klugen Staatsmann und tapferen Feldherrn Perikles zu Athens größten Zeiten mit dem völlig ungeschmückten, aber mit einem zweiten Gesicht samt Nase und Augen versehenen, furchterregenden,

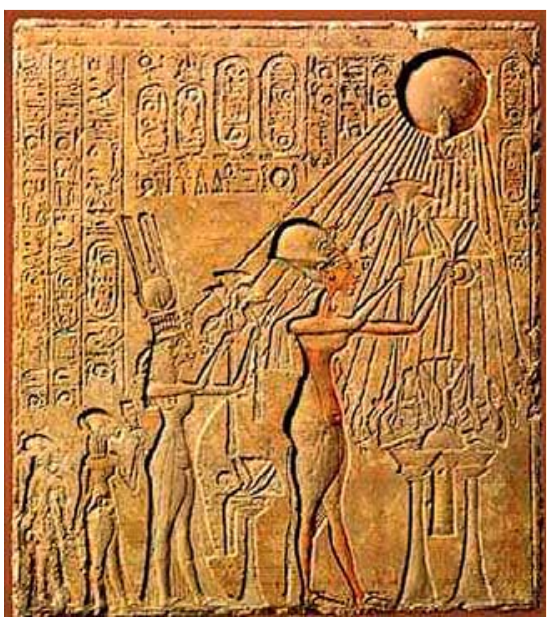

Abb. 6 Der seinen Sonnengott anbetende Pharao Echn-Aton. Der dolichozephal deformierte Kopf verschmilzt mit der kahlen Krone Oberägyptens zu einem riesigen, von der Sonne maximal beleuchteten und ihr ebenbürtigen glatzköpfigen Planeten (Bild: Wikipedia).

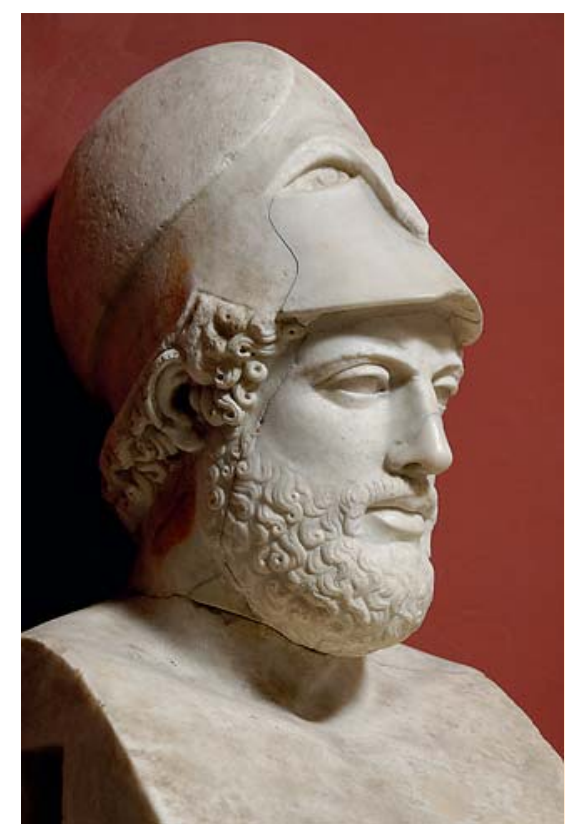

Abb. 7 Der Helm des Perikles, kahl und ungeschmückt, gleichsam ein zweiter Schädel mit zweitem Gesicht, Rom, Vatikanische Museen (Bild: Wikipedia, Marie-Lan Nguyen).

glatzenartigen Helm ohne Federbusch zeigen ( $\bullet$ Abb. 7). Helm und (kahler) Schädel bilden im Griechischen ein Wortspiel, da kranos der Helm und kranion der Schädel, also eigentlich „Helmchen“ heißt. Im Zusammenhang mit „Helm“ müssen wir noch einmal auf den griechischen Begriff für Kahlheit, nämlich phalakra, zurückkommen. Akra (wie z.B. in Akromegalie) heißt die Spitze, der Gipfel, die Extremität, hier für (kahle) Erhebung. Phalos ist ein bügelartiger, den Helm dorsal umgebender Wulst, sodass sich zwanglos der Vergleich mit einer androgenetischen Alopezie samt gut ausgeprägtem, wulstartigem, dorsalem „bügelartigem" Haarkranz ergibt. Genauso sind auch die Glatzen von Silenos und des Satyrn auf den antiken Schalen dargestellt. Sehr bemerkenswert, dass der Christ Synesios nicht auf die Stelle Epheser 6, 17 zu sprechen kommt, wo vom „Helm des Heils“ die Rede ist. Vielleicht liegt es daran, dass hier nicht das Wort kranos, sondern das sehr viel ungenauere perikephaleia gebraucht wird, das eigentlich nur Kopfbedeckung heißt. 


\section{Dionysos und Sokrates im Vergleich}

\section{$\nabla$}

Wir kommen zu Dionysos, Sokrates und Silenos. Wie oben schon ausgeführt, ist eine starke, dunkle, vielleicht sogar durch Tierfelle verstärkte Behaarung ein wildes, animalisches Attribut des Dionysos und Ausdruck eines in absolut dunkler Vergangenheit geprägten kulturellen Gedächtnisses (viele Abbildungen bei Wikipedia). In starkem Kontrast dazu beispielhaft die Philosophen Diogenes ( $\bullet$ Abb. 3) und vor allem Sokrates ( $\bullet$ Abb. 2). Sein kahler Kopf und seine auch im Übrigen durch die manchmal als syphilitisch beschriebene Sattelnase hässliche Physiognomie ist bereits durch vage Andeutungen von Zeitzeugen als einigermaßen realistisch einzuschätzen. Er ist mit seiner „Leuchtkraft“ (siehe homerischer Odysseus) ein Sinnbild für exaktes Denken, rationale Philosophie und Sophistik. Die dunkle, teils tierische Behaarung des Dionysos und die helle, leuchtende Glatze des Sokrates stehen sich gegenüber wie Dunkelheit und Licht, wie dionysischer, verschleierter Mythos einerseits und klarer, aufgeklärter Logos andererseits.

\section{Friedrich Nietzsche und die Geburt der Tragödie trichologisch betrachtet \\ $\nabla$}

Dieser kulturgeschichtliche, auch in der Trichologie frappant zum Ausdruck kommende Dualismus zwischen Dionysos und Sokrates war eines der Themen, die Friedrich Nietzsche in seinem Erstlingswerk „Die Geburt der Tragödie“ [6] aufgegriffen hat. Der dunkle, in immer wieder geübten orgiastischen Ausschweifungen alkoholischer und sexueller Natur sich ergehende Dionysoskult führte schließlich bei seinen Adepten zu Gefühlen des Ekels und des Überdrusses, sodann zum Nachdenken über schuldhaft verursachte existenzielle Probleme des Menschen, und damit allmählich in normativer Inversion zu einer der stärksten kulturellen Leistungen Griechenlands, nämlich der Geburt der Tragödie. Im Wort „Tragödie“ (Bocksgesang) ist das schamlose dionysische Treiben der bocksfüßigen Satyrn noch erhalten. Das Satyrspiel beendete bei den Festspielen stets einen Zyklus von Tragödienaufführungen.

Nietzsche lässt in seiner Schrift eindeutig erkennen, wem seine Sympathien gelten, nämlich nicht der klaren, philosophisch-deterministischen, eher sterilen Gedankenwelt des kahlköpfigen Tragödienverächters Sokrates, sondern der schöpferischen, dynamisch-expansiven, dunklen, in den schicksalhaften Verstrickungen des Menschseins befangenen Welt des gut behaarten Dionysos, dies ist auch an seinem eigenen, in frühen Jahren durchaus noch vorhandenen, die Nacht und Liebe von „Tristan und Isolde“ (so z. B. [6], S. 136) beschwörenden, spätromantischen Lebensgefühl erkennbar. Seine eigene Physiognomie mit dem kräftigen Haupthaar, den buschigen Augenbrauen und dem mächtigen Seehundsbart ist eindeutig dionysisch und nicht sokratisch (৫ Abb. 8).

Die Figur des Dionysos hat Nietzsche sein Leben lang nicht losgelassen, später kommt er mit den Dionysosdithyramben, in denen von allen existenziellen Zuständen des Menschen wie Liebe, Leid, Nacht und Tod die Rede ist, wieder auf ihn zurück. Nietzsche wäre, hätte er sich mit Synesios auseinander gesetzt, ganz gewiss als einer seiner prominentesten Kritiker aufgetreten.

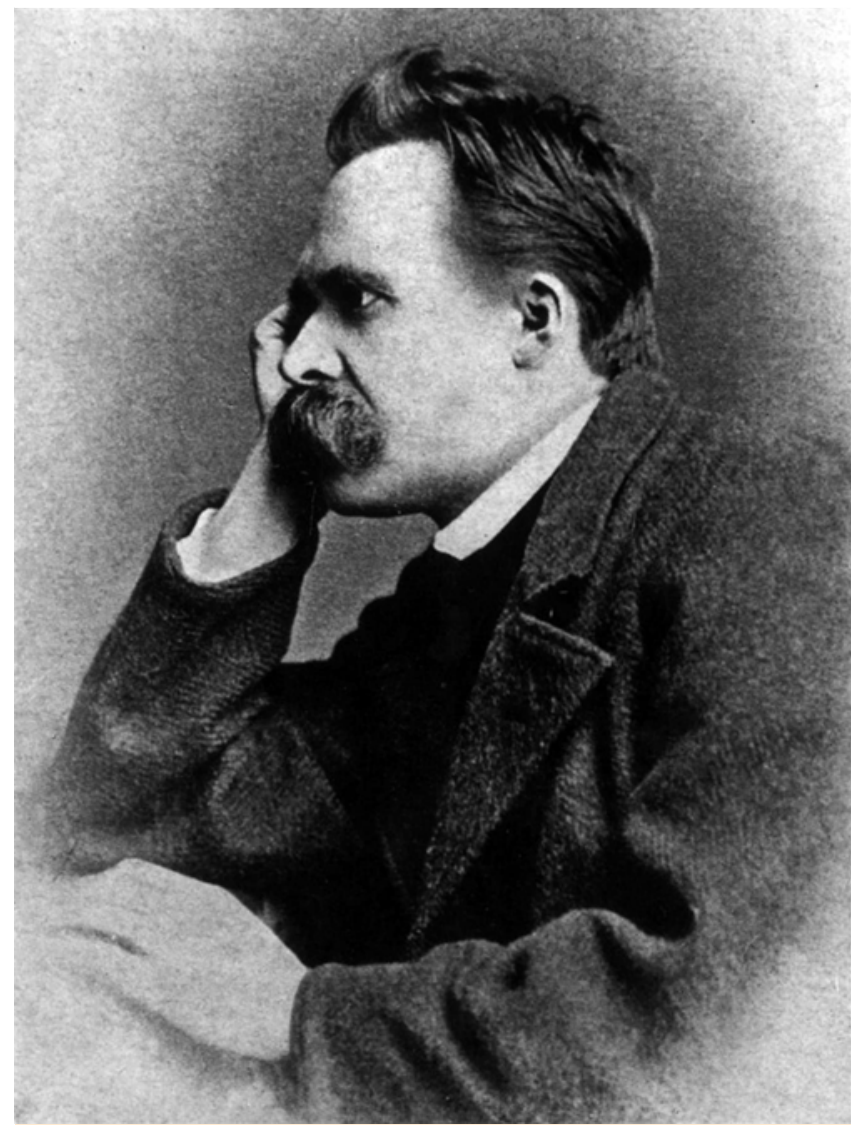

Abb. 8 Der deutsche Philosoph, Pamphletist und Autor der „Geburt der Tragödie“ Friedrich Nietzsche. Volles Haupthaar, buschige Augenbrauen und mächtiger Seehundsbart zeigen ein „dionysisches“ und kein „sokratisches" Wesen (Bild: Wikipedia).

\section{Darstellungen des Silenos}

\section{$\nabla$}

Zwischen Dionysos und Sokrates vermittelnd ist die Figur des mythengeschichtlich sicher jüngeren Silenos, des Zuchtmeisters des Dionysos angesiedelt. Der trichologische und endokrinologische Befund bei Silenos ist auf einer von Onesimos (tätig um 500-470 v. Chr. in Athen) bemalten Schale eindeutig (Abbildungen im Internet: Onesimos with Silenos). Das untere Stockwerk ist mit einem erigierten Penis „phallisch-dionysisch“ und zeigt eine erwünschte Androgenwirkung, während auf dem Kopf bei sehr starkem Bart- und Haarkranzwuchs eine deutliche androgenetische Alopezie zu sehen ist, das Zeichen einer „sokratischen“ Glatze, wenn auch mit Efeu bedeckt. Diese Androgenwirkung ist nach unserem heutigen Geschmack unerwünscht, die Dermatologie hat bis heute dagegen noch kein Patentrezept.

Auch das berühmte Bild von P. P. Rubens „Der trunkene Silen“ in der Münchener Alten Pinakothek ( $\bullet$ Abb.9) zeigt einen glatzköpfigen, dabei korpulenten, hässlichen alten Mann, Zeichen erwünschter Androgenwirkungen sind nicht mehr zu erkennen.

Die abgesehen vom Bart relativ schwache Körperbehaarung mit Bauchglatze und gerade noch erkennbarer, eher weiblicher Schamhaarbegrenzung lässt in Verbindung mit der Trunksucht sogar an einen Leberschaden mit relativem Oestrogenüberschuss denken. Nach dem Kommentar im Katalog der Gemäldesammlung handelt es sich mit großer Wahrscheinlichkeit um die unverblümte Darstellung des hässlichen Sokrates, wobei sein Abbild allerdings eher als Warnung vor den Folgen der Trunksucht, denn als Verherrlichung dionysischer Rauschzustände verstanden wer- 


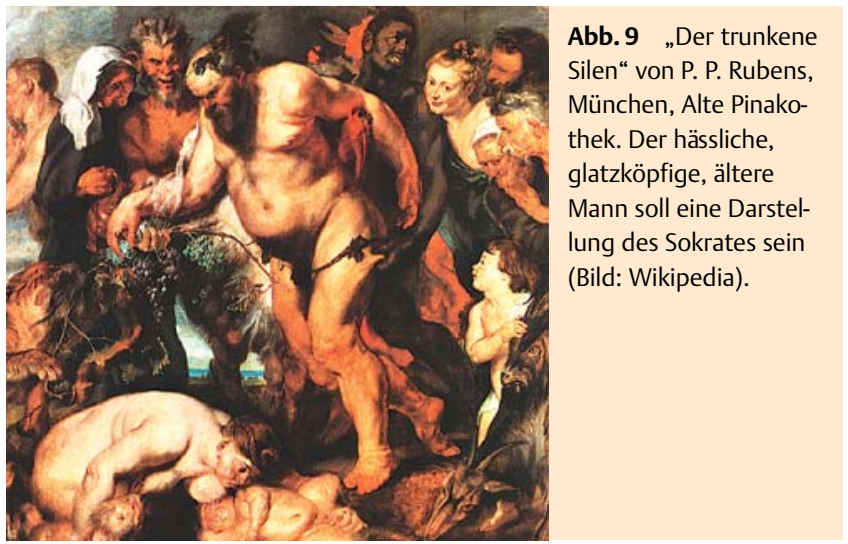

den soll ([7], S. 453-454). Nach [1], S. 29 hat Sokrates - auf sein Äußeres überhaupt wenig bedacht - merkwürdigerweise sich selbst mit Silenos verglichen, wollte aber sein „(kahles) Haupt zum Schatzhaus des Geistes machen“ ([1], S. 29). In einer Fußnote auf derselben Seite weist der Übersetzer Werner Golder auf eine Stelle in Platons „Symposion“ hin, in der Alkibiades seinen Lehrer mit bekannten Silenskulpturen vergleicht. Diese brauche man in der Mitte nur zu öffnen, um Götterbilder zu erkennen. Der Wein des Silenos am Ende also doch ein Zugang zu den Göttern?

\section{Wertigkeit der Glatze in der europäischen Geschichte $\nabla$}

Wir haben gesehen, dass konträr zu unserem heutigen Geschmack die Glatze im Altertum, vor allem in Ägypten und in Griechenland ein teilweise großes Ansehen genoss. Das hat sich in den vielen Jahrhunderten seitdem natürlich sehr verändert. Inspiriert von der Vergangenheit wollen wir die Entwicklung kurz betrachten.

Bereits in römischer Zeit scheint die Glatze wieder überwiegend negativ besetzt, die Caesaren werden in der Regel mit vollem lockigem Haar, der „coma caesarea“ als Ausdruck von Macht und Überlegenheit, abgebildet. Vor allem in Glyptotheken kann man sehr viele derartige Köpfe sehen. Das Mittelalter legte ebenfalls großen Wert auf eine volle Haarpracht, wie man beispielhaft an dem so bekannten Bamberger Reiter, dem das volle lockige Haar unter dem Stirnreif hervorquillt, erkennen kann. Für die frühmittelalterliche heilige Klothilde z.B. war die volle Haarpracht so wichtig, dass die ihnen drohende klösterliche Tonsur ein Grund war, ihre Enkel umzubringen. Die Geistesgrößen der Renaissance sind zur Kaschierung ihrer bestimmt nicht immer reichlichen Kopfbehaarung stets mit einer typischen Gelehrtenmütze dargestellt, so z.B. viele Abbildungen des Erasmus von Rotterdam. In der Barockzeit bediente man sich sogar der Perücke, wie aus vielen Darstellungen weltlicher und geistiger Größen bekannt. Mit Anbruch eines bürgerlichen Zeitalters begünstigt durch Aufklärung und Rationalismus bekannte man sich allmählich wieder offen zu natürlichen Verhältnissen und schämte sich seiner Kahlköpfigkeit nicht. Das Porträt Immanuel Kants mit Halbglatze und Teilperücke ( $\bullet$ Abb. 10) repräsentiert den Übergang vom feudalistischen zum bürgerlichen Zeitalter. Kulturgeschichtliche, philosophische, astrale, kosmologische und sakrale Kategorien und Analogien spielen seitdem bei der Gestaltung der Haartracht und Einschätzung der Glatze keine Rolle mehr.

Die Haartracht wird nur noch von utilitaristischen und am Zeitgeschmack orientierten ästhetischen Kategorien bestimmt, der-

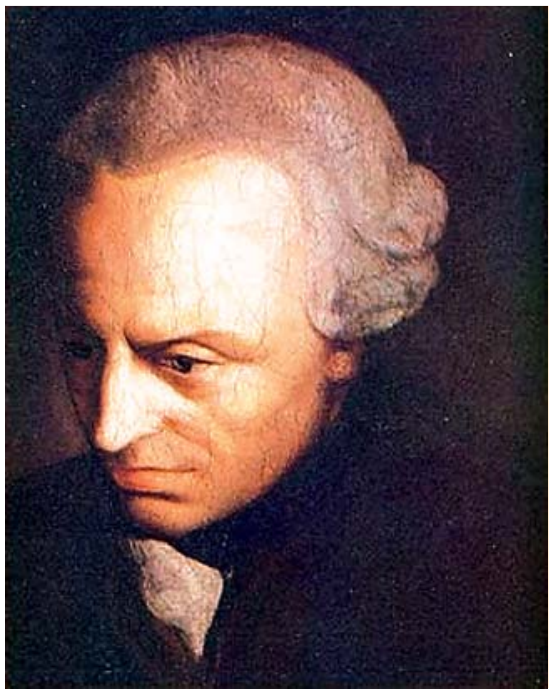

Abb. 10 Am Übergang zur Neuzeit steht das Porträt Immanuel Kants. Mit der Halbglatze und der Halbperücke wird ein Kompromiss zwischen Künstlichkeit und Natürlichkeit gefunden (Bild: Wikipedia). zeit ist immer noch schönes volles Haar bis ins hohe Alter die Wunschvorstellung.

\section{Blick auf Gegenwart und Zukunft}

Wenn nicht alles täuscht, sind jedoch künstliche Haarteile bei Männern mit Ausnahme von manchen Personen des öffentlichen Lebens unmodern geworden, die Wertigkeit der Glatze hat wieder zugenommen, ganze Fotogalerien im Internet belegen es. Diese Personen mit ihrem Kahlkopf erheben Anspruch auf Dominanz, Extravaganz und exklusives Außenseitertum. Auffallend ist auch das größer gewordene Bedürfnis nach Rasur oder Epilation, nicht nur des Kopfes - viele Glatzenträger rasieren den verbliebenen Haarkranz und unterstreichen damit ihre Seriosität -, sondern auch der übrigen Körperbehaarung, insbesondere der Achsel- und Schamhaare bei Männern und Frauen. Positiv zu beurteilen ist die zunehmende Toleranz gegenüber Glatzenträgern und man weiß auch öfters, dass manchmal eine Krankheit wie die totale Alopecia areata dahinter steckt. Der daran erkrankte, vor wenigen Jahren zurückgetretene, sehr bekannte italienische Fußballschiedsrichter Collina wäre noch vor 50-60 Jahren ohne Perücke gnadenlos verspottet worden und hätte auf dem Spielfeld keine Autorität genossen. Kürzlich sah ich in einer überfüllten Kirche aus allen Menschen „homerisch“ herausleuchtend, noch vor wenigen Jahren unvorstellbar, eine sonst sehr bürgerlich wirkende, noch junge Frau (!) mit totaler Alopezie ohne Perücke, bemerkenswert auch deshalb, weil diese in solchen schweren Fällen ja von der Krankenkasse bezahlt wird. Wer weiß, vielleicht wirkt auch die Clownsglatze in einigen Jahrzehnten oder Jahrhunderten nicht mehr lächerlich, sondern als etwas ganz Normales.

Werfen wir abschließend im Sinne des Synesios noch einmal einen Blick auf das Porträt Kants ( $\bullet$ Abb. 10), so sind wir überrascht, welche intellektuelle Kraft, welch „homerisches“ Leuchten und esoterischen Glanz der Maler der Kahlheit abgewinnen konnte. Viele Dermatologen werden auch Abbildungen des kahlköpfigen Gottfried Benn (sehr zahlreich z.B. bei Wikipedia im Internet) kennen, bei denen dem Fotografen ein ganz ähnlicher 
Effekt gelungen ist. Hatte man vielleicht unbewusst eine Rückprojektion auf den „Archetyp“ von Weisheit und Klugheit vor Augen?

\section{Danksagung}

Die brillante deutsche Übersetzung von „Lob der Kahlheit“ verdanke ich Werner Golder [1]. Aus dieser Übersetzung stammen auch alle wörtlichen und indirekten Zitate, sowie Hinweise zu Leben und Werk des Synesios von Kyrene und auf die klassischen griechischen Autoren.

\section{Abstract}

\section{On the „Praise of Baldness“ by Synesius of Cyrene with a Trichological Look Back to Antiquity and a Look Forward into the Present and Future $\nabla$}

The life and work of Synesius of Cyrene will be dealt with first, followed by important aspects of hairstyle in general. Then Synesius' most important arguments in praise of baldness from biology, cosmology, Greek mythology and contemporary history will be put forward. Partly by means of sophistic methods, the advantages of baldness are presented, especially by the example of demigod Asclepius, or his Egyptian model Imhotep as well as by the example of the bald philosopher Socrates in the context of the mythological figures of Dionysus and Silenus. The iconography of these figures and my own thoughts lead to surprisingly corroborative results. There will be references to Friedrich Nietzsche's early work „The Birth of Tragedy“ and its completely different judgement of Dionysus and Socrates as well as their analogy from a trichological point of view. On the whole it becomes evident that hairstyle and the appreciation of baldness are subject to change in the course of history. An even greater social acceptance of baldness is likely to occur in the future.

\section{Literatur}

1 Synesios von Kyrene. Lob der Kahlheit. Deutsch von Werner Golder Würzburg: Königshausen und Neumann; 2. Auflage 2007

2 Ogonczyk-Zakrzewski MF Ritter von. Medizinisch-literärische Geschichte des Weichselzopfes, ein Versuch. Wien: Mechitaristen-Congregations-Buchhandlung; 1830; 10

3 Möhn R. Der Weichselzopf - Krankheit, Aberglaube, Charakterprofil und Erinnerungskultur. Akt Dermatol 2008; 34: 49

4 Saueressig H. Martin Gumpert. Im Winkel der Medizingeschichte. In: Ärzte und Ärztliches. Essayistische Anregungen. Biberach/Riss: Basotherm $\mathrm{GmbH}$, Wege und Gestalten; 1989: 31

5 Mann TH. Joseph der Ernährer. In: Joseph und seine Brüder. Berlin: Deutsche Buchgemeinschaft; 1964: 970

6 Nietzsche F. Die Geburt der Tragödie oder Griechentum und Pessimismus. Köln: Könemann-Verlagsgesellschaft mbH; 1994: 6-151

7 UK. In: Katalog Alte Pinakothek München, Bayerische Staatsgemäldesammlungen 1983: $453-454$ 